Respuestas

Año 17

No. 1

Junio 2012

ISSN 0122-820X

PP: $48-54$

\title{
Multi-agent Model for Evaluation of Learning Objects from Repository Federations - ELO-index
}

\author{
Valentina Tabares $^{1} \mid$ Paula Rodríguez $^{1} \mid$ Néstor Duque $^{2}$ | Rosa Vicari ${ }^{3}$ | Julián Moreno
}

Recibido:

Diciembre 10 de 2011

Aceptado:

Abril 23 de 2012

\section{Abstract.}

Availability and reusability are desired characteristics in order to guarantee the quality of Learning Objects (LO) and, because of that, the implementation of metrics for these characteristics is important for their evaluation.

This paper describes an approach that uses a Multi-Agent System for assessing the LO, applying different methods and metrics and finally weighing them to obtain an index called ELO-index. Using metadata as our source of information, the metrics used for calculating ELO-index was completeness, consistency and coherency.

The obtained index can be used to recommend LO by matching them with user-provided keywords, but also to manage the repository in which they are stored evaluating their quality before being published.

Keywords: Learning objects evaluation, Metrics, Multi-agent System

\section{Introduction}

${ }^{1}$ Universidad Nacional de Colombia Sede Medellín vtabaresm@unal.edu.co, parodriguezma@unal.edu.co, jmoreno1@unal.edu.co

${ }^{2}$ Universidad Nacional de Colombia Sede Manizales ndduqueme@unal.edu.co

${ }^{3}$ Universidade Federal do Rio Grande do Sul rosa@inf.ufrgs.br
7 oday there are many Learning Objects Repositories which allows for searching and retrieving information. Unfortunately, they only allow for users to choose their interests among terms, which are often inadequate to express what users are really interested in, or the obtained results are not the desired.

"The main functionality of a digital repository, to provide access to resources, can be severely affected by the quality of the metadata. The resource will just be part of the repository but will never be retrieved in relevant searches. The usefulness of a digital repository is strongly 
correlated to the quality of the metadata that describe its resources" [1]. Metadata is used to describe and identify educational resources. These data facilitate the search, retrieval and selection of LO, allowing re-use, one of its greatest challenges [2].

According to [3], the growth in the number, size and diversity of digital collections makes metadata quality an increasingly important issue. Consequently, appropriate software tools offer great potential for collection managers to analyze their repositories and verify that their metadata supports their users' interactions [3].

In [4], as the result of their study, authors indicate that inaccurate, incomplete and inconsistent metadata is usually a common issue in repositories. This imply a problem for resource sharing and access across digital collections. In [5], an experimental study is presented with university students oriented to assess the LO obtained from specific searches evaluating response time and the number of the retrieved resources. In this case, relevance was measured by various parameters (effectiveness of content, motivation for learn and easy for use). The results showed that in the pedagogical aspect, most LO obtained are not suited to the objectives pursued, nor for motivated learning. In terms of usability, the matter is not very encouraging since the ratings are very low.

In order to detect these problems and propose some solutions, several researches have been focused on different topics. Some of them aimed to evaluate LO from the pedagogical point of view considering the content structure, whereas another ones focused only in the the metadata. The research presented on this paper is located in the second group and its goal refers to the assessing of the quality of the metadata in the repositories.

The authors in [6] proposes metrics based on the same quality parameters used for human review of metadata: completeness, accuracy, provenance, conformance to expectations, logical consistency and coherence, timeliness, and accessibility. The information requirements to calculate the proposed metrics are also detailed. Early results suggest that the metrics are indeed sensitive to quality features in the metadata. Finally, such a research recommends further work to validate and calibrate the proposed metrics.

The work in [7] propose a new conceptual framework that defines metadata quality in terms of its fundamental components, namely: correctness, completeness and relevance. It implied logic rules, which include, impose or prohibit certain values in the fields of a metadata record. In [8], authors describe the design and implementation of an infrastructure to support metadata quality assessment that evaluate the semantic and syntactic content of metadata from a qualitative perspective. A Web-based metadata quality tool is proposed in [3]. This tool provides statistical descriptions and visualizations of metadata. It detects metadata quality issues to guarantee compliance with the requirements.

We define in this article an index called the ELO-index, and present an approach based on a Multi-Agent System for assessing the LO, applying different methods and metrics joint to a weighing factor. Our source of information is the metadata and the metrics used for calculating ELO-index are completeness, logical consistency and coherence. The selection of metadata and weights were obtained from consultation with experts in different repositories and specialized users. ELO-index can be used, on the one hand, for recommendation of the objects that match the user-provided key-words and on the other hand, for management of the repository prior to publishing and after evaluating the quality of the objects stored. This last issue is validated with a case study. 


\section{Metrics}

In the domain of the LOs quality is understood as a set of criteria for evaluating ed-ucational resources and determines their level of relevance in the teaching-learning processes [9]. There are many aspects that must be reviewed to determine quality, one of the most important are metadata. In fact it could be said that the usefulness of a repository depend on such metadata because they are responsible for describing all resources.

Completeness indicates if the metadata describes the objects as much as possible making it available for use. This allows for verifying if each instance provides information for the detailed description of the resource and measures how much information is available about the resource. The quality of metadata declines with the absence of core elements which have been used for search and recovery. Consistency estimates the level of compliance with the metadata standard or the rules established by the repository that stores them. It can occur when you include metadata defined in the standard, when the metadata includes values that are not within the options. Coherence implies the level to which the LO metadata describe the same resource [8] [10].

\subsection{Completeness Metric}

In order to determine the completeness of an object metadata we propose to use a metric which verifies that fields are fulfilled.

50 Metadata standards such as LOM, Dublin Core and OBAA specify a basic outline of metadata that must be filled out to describe an LO. To verify its completeness, metadata could be reviewed field by field determining if they contain any value and in the case of multi-valued fields if there is at least one instance.

Most of the repositories only take into account some of the metadata for search, therefore it is not necessary that all fields are fulfilled, only the most used. As concluded in the work of [11], the mainly used is the general information, the information associated with the life cycle and the educational characteristics of $\mathrm{LO}$.

Researches like [8], [4] and [1] verify the completeness of metadata evaluating only some fields, which are considered the "most frequently employed".

In our proposal the following metadata were selected. However, considering that not all of them have the same relevance, we assigned a weighting factor. This factor represents the importance of the field and the sum of all is equal to 1 . One of the criteria used to select them were the considered fields during a search in the Federação Educa Brasil (FEB).

Table 1. Weighing Factor

\begin{tabular}{|c|c|}
\hline Metadata i & Factor ki \\
\hline Title & 0.17 \\
\hline Language & 0.05 \\
\hline Description & 0.15 \\
\hline Keywords & 0.13 \\
\hline Author & 0.09 \\
\hline Date & 0.06 \\
\hline Format & 0.08 \\
\hline Localization & 0.12 \\
\hline Learning Resource Type & 0.05 \\
\hline Interactivity Type & 0.05 \\
\hline Aggregation Level & 0.05 \\
\hline
\end{tabular}

For calculating the metric, a value of 1 is assigned if the metadata have value (not null) and 0 for other case, then such a value is multiplied for the corresponding factor according to table 1 . The accumulate result is the metric value and its range is $[0,1]$. A value of 1 means that LO is fully complete, whereas a value of 0 means that $\mathrm{LO}$ is completely metadata empty. The corresponding formula is:

$$
\text { ConsisMetric }=1-\sum_{1}^{n}(M i) / R
$$


Where:

is the Weighing Factor.

$\mathrm{Mi}=1$ if value of metadata is not null, $\mathrm{Mi}=0$ if value of metadata is null.

\subsection{Consistency Metric}

For estimating the consistency is necessary to determine the level of compliance with the metadata through rules according to recommendations for values in the standard. Table 2 shows some rules selected to calculate this metric:

For calculating this metric, a value of 1 is assigned if the metadata complies with rule and 0 is assigned otherwise. The accumulate result is the metric value and its range is $[0,1]$. A value of 1 means that $\mathrm{LO}$ is completely consistent, whereas a value of 0 means that $\mathrm{LO}$ is completely inconsistent. The corresponding formula is:

$$
\text { ConsisMetric }=1-\sum_{1}^{n}(M i) / R
$$

Where:

is the number of rules analyzed

$M i=1$ if complies with rule, $M i=0$ if not complies with rule

\subsection{Coherence Metric}

In order to determine the coherence between metadata is necessary to verify if the information contained in one field have correlation with others. So, the semantic distance is calculated between the different free text fields.

To calculate the semantic distance the cosine measure is proposed, which measures the similarity between arrays. In this case such arrays are the words contained in the description of the metadata. We selected Title, Description and Keywords fields due to its importance in the description of LOs.

$$
\text { CohereMetric }=\sum_{1}^{k} \sum_{1}^{n}(P i * Q i) / \sqrt{\left(\sum_{1}^{n} P i^{2} * \sum_{1}^{n} Q i^{2}\right)} / K
$$

Where:

is the number of metadata analyzed $P i$ is term frequency $i$ in field 1 Q $i$ is term frequency $i$ in field 2

\section{Multi-agent System Development}

This section shows some aspects of the modelling phase of the Multi Agent System following the MAS-CommonKADS methodology [12], emphasizing on the different components of the system. MASCommonKADS, extends the knowledge engineering methodology CommonKADS

Table 2. Rules to check consistency

\begin{tabular}{|c|l|}
\hline $\begin{array}{c}\text { Rule 1: } \\
\text { Value Learning } \\
\text { ResourceType }\end{array}$ & $\begin{array}{l}\text { Valid values=Exercise, simulation, questionnaire, diagram, figure, graph, } \\
\text { index, slide, table, narrative text, exam, experiment, problem statement, self } \\
\text { assessment, lecture }\end{array}$ \\
\hline & Valid values=Author, Publisher, Unknown, Initiator, Terminator, Validator, \\
Rule 2: & Editor, Graphical, Designer, Technical Implementer, Content Provider, \\
Value Role & Technical Validator, Educational Validator, Script Writer, Instructional \\
& Designer, Subject Matter Expert \\
\hline Rule 3: & If Structure=atomic then AggregationLevel=1 \\
Structure vs. & If Structure=collection then Aggregation Level=2 \\
AggregationLe & If Structure=networked then Aggregation Level=3 \\
vel & If Structure=hierarchical then Aggregation Level=4 \\
\hline If Structure=linear then Aggregation Level=4
\end{tabular}


Respuestas

Año 17

No. 1

Junio 2012

ISSN 0122-820X with techniques from object-oriented and protocol engineering methodologies, and defines the necessary models for the analysis and design phases providing a complete documentation.

For the description of the tasks that require knowledge the control that determines how the elemental inferences are integrated is specified in order to calculate the ELO-index, this is showed in Figure 1.

The description of the conversations among agents is made both in graphical and textual representations as shown in Figure 2.

A complete diagram depicting the proposed system is presented in Figure 3.
The Evaluator Agent is responsible for the ELO-Index calculation and takes into account the values obtained in the three metrics and the weight assigned previously. This agent has a deliberative nature since after receiving information of the completeness, consistency and coherence agents, performs the calculation of the weighted single evaluation for each LO.

The metrics agents receive the LO metadata and calculate the corresponding metric applying the formulas previously described. These agents are:

Completeness Agent: This agent performs a review of the metadata after communicating

Fig. 1. Task Model

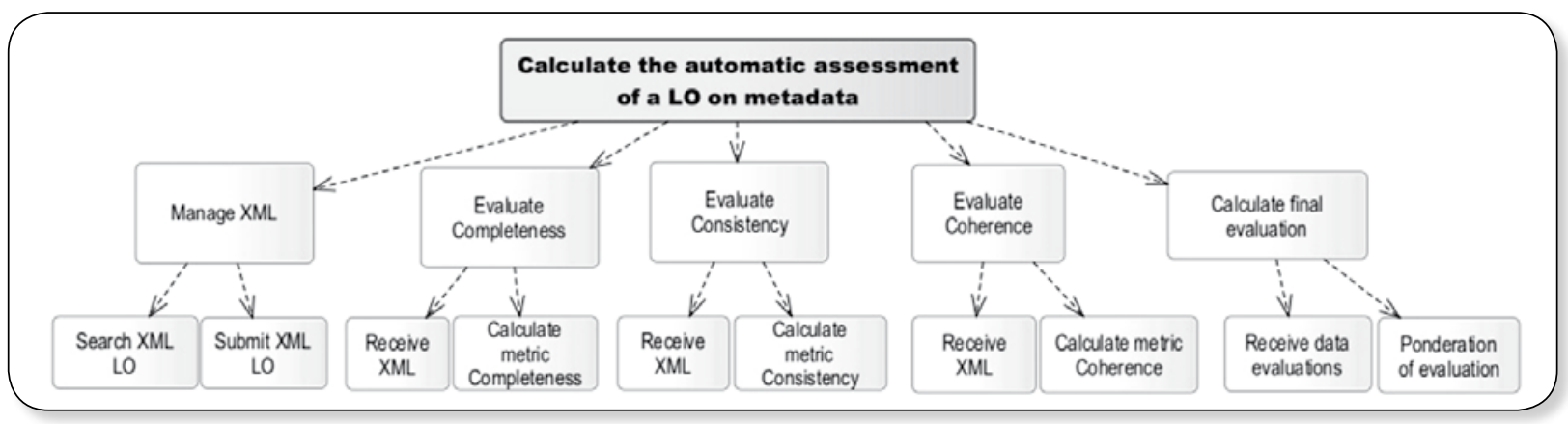

Fig. 2. Sequence Model

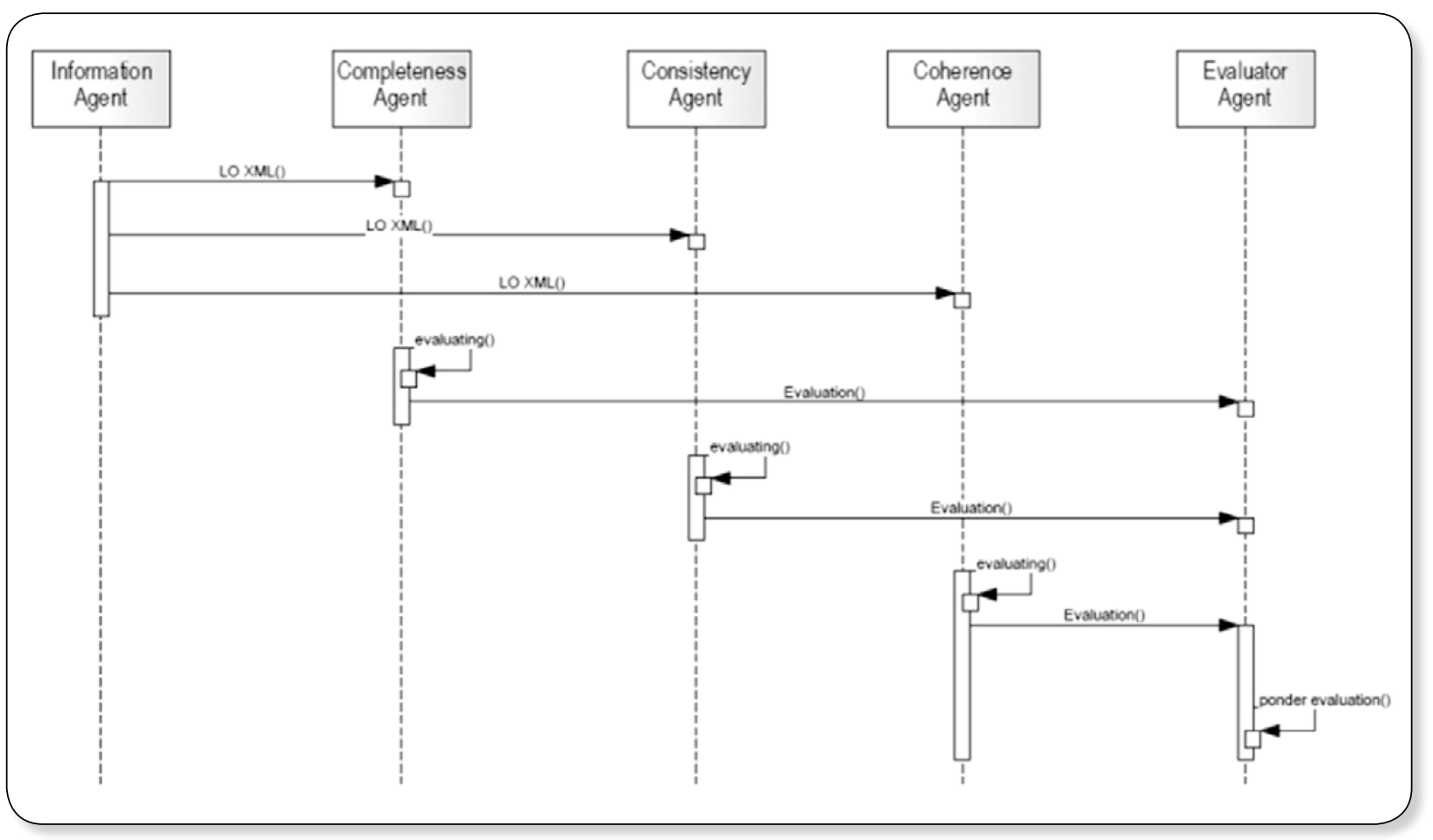


with an information agent to receive the XML. Later it calculates the completeness metric using the weights associated with the fields which are stored in its knowledge base.

Consistency Agent: The behavior of this agent includes the implementation of rules intended to assess compliance with the standard metadata specific to the LO. More specifically, it compares with expected values specified in the standard OBAA.

Coherence Agent: This agent implements the behavior Coherence, which applies a filter to remove excess words and / or irrelevant words on the content of three specific fields: title, keywords and description. Later it makes the calculation of the cosine similarity among these 3 fields to verify the relationship between the terms.

Fig. 3. Multi-agent System Architecture

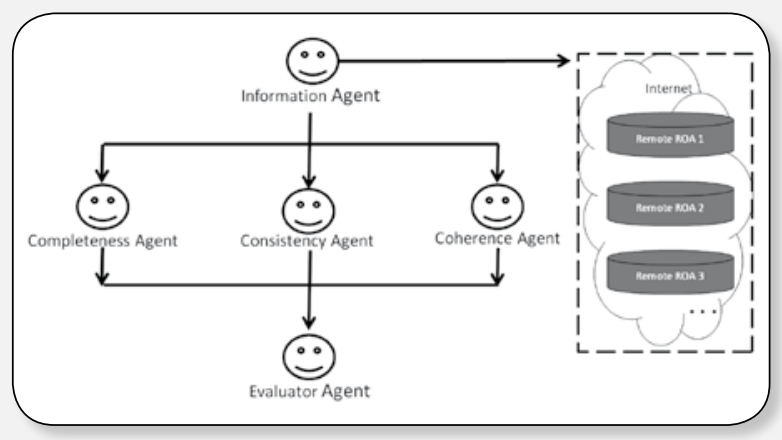

Information Agent: This agent is responsible of retrieving and managing the XML metadata for LO stored in the remote repository and federations. This agent communicates with the agents who develop evaluation algorithms to deliver the XML. The evaluation mechanism is based on the metadata that describes the LO, permitting to use diverse visions for the resource composition, naming and allocation, and supporting several standard technologies.

\section{Experimental Work}

The validation of the proposal was made over LO obtained of repositories in the Federation Educa Brazil, FEB. The data was obtained randomly through of the OAIPMH interface. One collection of 100 OBAA records was tested. The metrics have been normalized to a 0 to 1 scale. The Table 3 and Figure 4 present a summary of the results.

Table 3. Evaluation Summary

\begin{tabular}{|c|c|c|c|c|}
\hline & Completeness & Consistency* & Coherence & ELO-index \\
\hline Average & 0,6764 & 0,4700 & 0,2446 & 0,4721 \\
\hline Mode & 0,6300 & 0,6000 & 0,3333 & 0,4107 \\
\hline Stdev & 0,0587 & 0,1691 & 0,1214 & 0,0760 \\
\hline Minimum & 0,5700 & 0,2000 & 0,0000 & 0,2880 \\
\hline Maximum & 0,7800 & 0,6000 & 0,5210 & 0,5883 \\
\hline $\begin{array}{c}\text { Confidence } \\
\text { level }(95,0 \%)\end{array}$ & 0,0116 & 0,0335 & 0,0241 & 0,0151 \\
\hline \multicolumn{7}{|l}{} \\
\hline
\end{tabular}

*The calculation of the metric consistency is affected by the values that not exist in metadata. Null values format $=37$, Structure = 99 and Aggregation Level, Interactivity Type and Interactivity Level $=100$.

Figure 4 shows the index values for each of the metric calculated in this work, completeness, consistency and coherence, also show the ELO-index metric; the index values are maintained in an unfavorable range below 0.6. The metric of completeness shows better values, while coherence metric has higher variability and lower values, which should generate concern about the recovery of $\mathrm{LOs}$ that are not expected.

Fig. 4. Evaluation Metrics and ELO-index

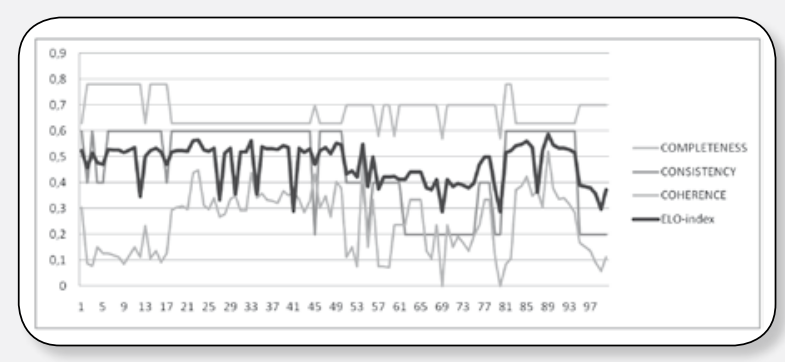

\section{Conclusion and}

\section{Future Work}

Applying the quality metrics proposed in this work allows for demonstrating that is necessary to evaluate the metadata of the evaluated repository. This is due to the 
obtained values reflect identifiable quality flaws, turning this way in a relevant tool for the repository management.

Modeling the problem using a MAS technique was an excellent option, allowed the disintegration into functional blocks, without losing the systemic point of view, which leads to distributing the solution in diverse entities that require specific knowledge, processing and communication between each other. The MAS allows the neutral vision in the proposed model.

As future work, a much more detailed validation of the metrics must be performed as well as a refinement of the underlying formulas. We also hope to calculate similarity semantic aspects using ontologies. In addition, we would like to implement a metadata translation tool that allows the use of different standards in a transparent form.

\section{Acknowledgements}

We are grateful with the group of the Federação de Repositórios Educa Brasil - FEB, in Porto Alegre, for their collaboration.

\section{References}

[1] X. Ochoa, "Learnometrics: Metrics for Learning Objects," Katholieke Universiteit Leuven, 2008.

[2] R. Vicari et al., "Proposta Brasileira de Metadados para Objetos de Aprendizagem Baseados em Agentes (OBAA)," Novas Tecnologias na Educação, 2010.

[3] D. Nichols, C.-hsiang Chan, and D. Bainbridge, "A tool for metadata analysis," 2008.

[4] Y. Bui and J.-ran Park, "An Assessment of Metadata Quality : A Case Study of the National Science Digital Library Metadata Repository," 2006.
[5] V. Tabares, N. Duque, and J. Moreno, "Análisis experimental de la utilidad en la recuperación de objetos de aprendizaje desde repositorios remotos," Sin Publicar, pp. 1-12, 2011.

[6] X. Ochoa and E. Duval, "Quality Metrics for Learning Object Metadata," Proceedings of World Conference on Educational Multimedia, Hypermedia and Telecommunications, pp. 1004-1011, 2006.

[7] T. Margaritopoulos, I. Mavridis, M. Margaritopoulos, and A. Manitsaris, "A Conceptual Framework for Metadata Quality Assessment," pp. 104-113, 2008.

[8] B. Hughes, "Metadata Quality Evaluation : Experience from the," pp. 320 329, 2004.

[9] E. Morales, A. Gil, and F. García, "Arquitectura para la Recuperación de Objetos de Aprendizaje de calidad en Repositorios Distribuidos," SCHA: Sistemas Hipermedia Colaborativos y Adaptativos. II Congreso Español de Informática CEDI 2007, vol. 1, pp. 3138, 2007.

[10] T. R. Bruce and D. I. Hillmann, "The Continuum of Metadata Quality: Defining, Expressing, Exploiting," Metadata in Practice, 2004.

[11] P. A. Rodríguez, G. Isaza, and N. D. Duque, "Búsqueda personalizada en Repositorios de Objetos de Aprendizaje a partir del perfil del estudiante," CAVA 2011, 2011.

[12] C. Á. Iglesias Fernández, "Definición de una Metodología para el Desarrollo de Sistemas Multiagentes," Universidad Politécnica de Madrid, 1998. 